\title{
Melting heat transfer in boundary layer stagnation-point flow towards a stretching/shrinking sheet.
}

\begin{abstract}
An analysis is carried out to study the steady two-dimensional stagnation-point flow and heat transfer from a warm, laminar liquid flow to a melting stretching/shrinking sheet. The governing partial differential equations are converted into ordinary differential equations by similarity transformation, before being solved numerically using the Runge-Kutta-Fehlberg method. Results for the skin friction coefficient, local Nusselt number, velocity profiles as well as temperature profiles are presented for different values of the governing parameters. Effects of the melting parameter, stretching/shrinking parameter and Prandtl number on the flow and heat transfer characteristics are thoroughly examined. Different from a stretching sheet, it is found that the solutions for a shrinking sheet are non-unique.
\end{abstract}

Keyword: Melting; Heat transfer; Stagnation-point flow; Stretching/shrinking sheet; Dual solutions. 